\title{
Distance Transforms on Anisotropic Surfaces for Surface Roughness Measurement
}

\author{
Leena Ikonen $^{1}$, Toni Kuparinen ${ }^{1}$, Eduardo Villanueva ${ }^{1}$, and Pekka Toivanen ${ }^{2}$ \\ ${ }^{1}$ Laboratory of Information Processing, Department of Information Technology \\ Lappeenranta University of Technology, P.O.Box 20, 53851 Lappeenranta, Finland \\ \{leena.ikonen, toni.kuparinen\}@lut.fi, villanue@gmail.com \\ 2 Tampere University of Technology / Digital Media Institute / EPANET \\ FRAMI, Kampusranta 9C, 60320 Seinäjoki, Finland \\ pekka.toivanen@tut.fi
}

\begin{abstract}
The Distance Transform on Curved Space (DTOCS) calculates distances along a gray-level height map surface. In this article, the DTOCS is generalized for surfaces represented as real altitude data in an anisotropic grid. The distance transform combined with a nearest neighbor transform produces a roughness map showing the average roughness of image regions in addition to one roughness value for the whole surface. The method has been tested on profilometer data measured on samples of different paper grades. The correlation between the new method and the arithmetic mean deviation of the roughness surface, $S_{a}$, for small wavelengths was strong for all tested paper sample sets, indicating that the DTOCS measures small scale surface roughness.
\end{abstract}

\section{Introduction}

The roughness of a surface is a property that needs to be measured in many applications, and the application motivating our research is in quality assessment of paper. Paper roughness has a significant effect on printability, which eventually defines the quality of the printed product. The roughness of paper is measured using profilometers, which acquire the real topography of a surface. The basic idea of profilometers is that they have a stylus, which travels on a surface measuring its height [1]. The Distance Transform on Curved Space (DTOCS) measures distances along surfaces represented as gray-level height maps, or range images, and can be used directly on the profilometer data. Here, the DTOCS and its locally Euclidean modification, the Weighted DTOCS (WDTOCS) [2] are generalized to anisotropic profilometer data, and used for estimating surface roughness based on the fact that distance values calculated along a highly varying surface are larger than distances calculated along a smoother surface. The presented method combines the DTOCS with the nearest neighbor transform (NNT), and produces a roughness map, which can be used to compare the roughness of different regions in the same image. In addition, an average roughness value can be calculated to characterize the whole surface. Other approaches to roughness inspection utilize statistical features, like kurtosis [3, roughness parameters, Fourier analysis [4, wavelets [5], and fractal dimension [6, 7]. 


\section{DTOCS for Anisotropic Grids}

The DTOCS calculates distances along gray-level surfaces, when gray-levels are understood as height values. Local distances, which are summed along digital paths to calculate the distance transform, are defined as $d\left(p_{i}, p_{i-1}\right)=\mid \mathcal{G}\left(p_{i}\right)-$ $\mathcal{G}\left(p_{i-1}\right) \mid+1$, where $\mathcal{G}(p)$ denotes the gray-value of pixel $p$, and $p_{i-1}$ and $p_{i}$ are subsequent pixels on a path. The WDTOCS produces more accurate distance values by using the locally Euclidean distance between pixels, and the Optimal DTOCS [8] improves the distance approximation even further. The DTOCS and the WDTOCS can be used for profilometer data consisting of real height values without any changes in the distance definitions. The integer gray-values defining the height differences are replaced with the floating point altitude data. Alternatively, the height data could be represented using units, which can be scaled to integers, for example, data represented in micrometers could be scaled by 10 and then rounded. However, the accuracy of the measuring device can be fully exploited by using the floating point data directly. Converting the data to a volume image and using well known distance transforms in 3D [9] would also require rounding of the height measurements, and result in an increased problem size.

The scaling of the surface must be carefully considered, when using the DTOCS. The values of the pixels represent the height, or the $z$-coordinate, of the surface represented as a range image. If the resolution in the $x y$-plane differs from the resolution in the $z$-direction, the height differences must be scaled in order to obtain approximations of true distances along the surface. Scaling in the horizontal image plane is needed, if the grid of the range image is anisotropic or rectangular, that is, if the resolution in the $x$-direction differs from the resolution in the $y$-direction. Interpolating additional values in the direction with the lower resolution would inevitably introduce some error compared to measured data, and lead to a multifold increase in the image size. Instead, the DTOCS local distances are generalized as follows:

$$
d\left(p_{i}, p_{i-1}\right)=\left\{\begin{array}{l}
r_{z}\left|\mathcal{G}\left(p_{i}\right)-\mathcal{G}\left(p_{i-1}\right)\right|+r_{x}, p_{i-1} \text { neighbor of } p_{i} \text { in } x \text {-dir. } \\
r_{z}\left|\mathcal{G}\left(p_{i}\right)-\mathcal{G}\left(p_{i-1}\right)\right|+r_{y}, p_{i-1} \text { neighbor of } p_{i} \text { in } y \text {-dir. } \\
r_{z}\left|\mathcal{G}\left(p_{i}\right)-\mathcal{G}\left(p_{i-1}\right)\right|+\max \left(r_{x}, r_{y}\right), p_{i-1} \text { diag. neighbor of } p_{i}
\end{array}\right.
$$

where $r_{z}$ is the scaling factor for the height differences, and $r_{x}$ and $r_{y}$ are the distances between neighbor pixels in the $x$ - and $y$-direction, as visualized in Fig. 1 The factors $r_{x}, r_{y}$ and $r_{z}$ may have any non-negative values, not necessarily integers. Similarly, the WDTOCS, in which the local distance is calculated using the Pythagoras' theorem from the height difference and the horizontal displacement between the neighbor pixels, can be generalized to rectangular grids as follows:

$$
d\left(p_{i}, p_{i-1}\right)=\left\{\begin{array}{l}
\sqrt{r_{z}^{2}\left|\mathcal{G}\left(p_{i}\right)-\mathcal{G}\left(p_{i-1}\right)\right|^{2}+r_{x}^{2}}, p_{i-1} \text { neighbor of } p_{i} \text { in } x \text {-dir. } \\
\sqrt{r_{z}^{2}\left|\mathcal{G}\left(p_{i}\right)-\mathcal{G}\left(p_{i-1}\right)\right|^{2}+r_{y}^{2}}, p_{i-1} \text { neighbor of } p_{i} \text { in } y \text {-dir. } \\
\sqrt{r_{z}^{2}\left|\mathcal{G}\left(p_{i}\right)-\mathcal{G}\left(p_{i-1}\right)\right|^{2}+r_{x}^{2}+r_{y}^{2}}, p_{i-1} \text { diag. neighbor of } p_{i}
\end{array}\right.
$$




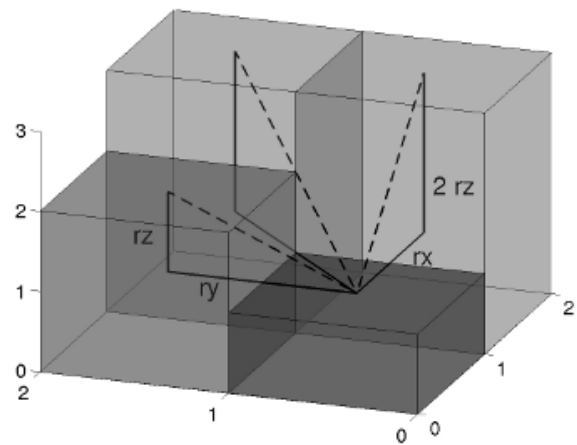

Fig. 1. Local distance definitions for the DTOCS (solid lines) and the WDTOCS (dashed lines) in an anisotropic grid visualized on a surface of $2 \times 2$ pixels

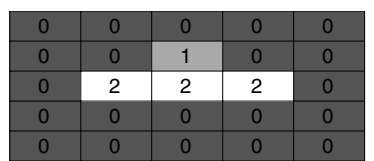

(a) Original image

\begin{tabular}{|l|l|l|l|l|}
\hline 3 & 1 & 3 & 6 & 9 \\
\hline 3 & 0 & 4 & 6 & 9 \\
\hline 3 & 3 & 5 & 8 & 9 \\
\hline 4 & 6 & 8 & 10 & 10 \\
\hline 5 & 7 & 9 & 11 & 11 \\
\hline
\end{tabular}

(b) DTOCS

\begin{tabular}{|l|l|l|l|l|}
\hline 3 & 1 & 3 & 6 & 9 \\
\hline 3 & 0 & 3 & 6 & 9 \\
\hline 3 & 1 & 3 & 6 & 9 \\
\hline 4 & 2 & 4 & 6 & 10 \\
\hline 5 & 7 & 5 & 7 & 11 \\
\hline
\end{tabular}

(d) Proj. dist. (DTOCS)

\begin{tabular}{|l|l|l|l|l|}
\hline 3.16 & 1.00 & 3.16 & 6.16 & 9.16 \\
\hline 3.00 & 0.00 & 3.16 & 6.32 & 9.32 \\
\hline 3.16 & 2.24 & 3.74 & 6.48 & 9.49 \\
\hline 4.16 & 4.47 & 5.98 & 7.48 & 10.22 \\
\hline 5.16 & 5.47 & 6.98 & 8.48 & 10.65 \\
\hline
\end{tabular}

(c) WDTOCS

\begin{tabular}{|l|l|l|l|l|}
\hline 3.16 & 1.00 & 3.16 & 6.16 & 9.16 \\
\hline 3.00 & 0.00 & 3.00 & 6.32 & 9.32 \\
\hline 3.16 & 1.00 & 3.16 & 6.16 & 9.49 \\
\hline 4.16 & 2.00 & 4.16 & 6.32 & 9.32 \\
\hline 5.16 & 3.00 & 5.16 & 7.32 & 9.49 \\
\hline
\end{tabular}

(e) Proj. dist. (WDTOCS)

Fig. 2. Example of the DTOCS, the WDTOCS and the corresponding projection distances in a rectangular grid, where $r_{x}=3$ and $r_{y}=1$

\begin{tabular}{|l|l|l|l|}
\hline 12.30 & 1.32 & 3.37 & 1.32 \\
\hline 7.91 & -0.44 & 4.25 & 1.61 \\
\hline 1.32 & 1.61 & 4.83 & 6.01 \\
\hline-1.61 & 7.76 & 6.15 & 8.79 \\
\hline 2.78 & 9.08 & 5.86 & 10.99 \\
\hline
\end{tabular}

(a) Original image

\begin{tabular}{|l|l|l|l|}
\hline 17.74 & 2.76 & 8.81 & 15.86 \\
\hline 13.35 & 0.00 & 9.69 & 15.57 \\
\hline 6.76 & 3.05 & 10.27 & 16.45 \\
\hline 10.69 & 10.20 & 12.59 & 19.23 \\
\hline 16.08 & 12.52 & 13.88 & 22.43 \\
\hline
\end{tabular}

(b) DTOCS

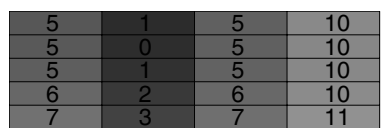

(d) Proj. dist (DTOCS)

\begin{tabular}{|l|l|l|l|}
\hline 13.73 & 2.02 & 6.36 & 11.77 \\
\hline 9.73 & 0.00 & 6.85 & 11.76 \\
\hline 5.39 & 2.28 & 7.34 & 12.25 \\
\hline 8.31 & 8.51 & 8.99 & 13.79 \\
\hline 12.82 & 10.17 & 10.03 & 16.02 \\
\hline
\end{tabular}

(c) WDTOCS

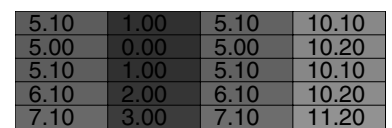

(e) Proj. dist (WDTOCS)

Fig. 3. Example of the DTOCS, the WDTOCS, and the corresponding projection distances on anisotropic profilometer data, where $r_{x}=5$ and $r_{y}=1$ 
A small example, where the step lengths are $r_{x}=3$ and $r_{y}=1$, and the height scaling is $r_{z}=1$, is shown in Fig. 2. The DTOCS and the WDTOCS calculated from one reference pixel in the rectangular grid are shown in Fig. 2 (b) and (c). Fig. 3 demonstrates that the DTOCS and the WDTOCS can be applied also to images with elongated pixels with floating point values. It can be seen that the DTOCS produces significantly larger values than the WDTOCS. Adding the horizontal and vertical displacement in the local distance definition clearly overestimates the locally Euclidean distance, when the values of neighbor pixels differ by several units.

The DTOCS and its generalization to anisotropic grids are metrics, that is, the distances are symmetric, positive definite and fulfill the triangle inequality, as long as only integer gray-levels and scaling factors are involved [10]. Calculating floating point distance values, either by using the WDTOCS definition or by having floating point input data, may result in violations of the metrics criteria, due to the limited precision available. For example, the least significant bits of the distance values calculated from pixel $p$ to pixel $q$ and from $q$ to $p$ may differ, as they are the result of several floating point operations.

The roughness measurement method utilizes normalized distance values. Distances are divided by the so called projection distance [11. The projection distance value of pixel $p$ is the length of the shortest path from $p$ to the nearest reference pixel projected into the image plane. The local distances are obtained by removing the effect of the height differences from the DTOCS or WDTOCS local distances, that is, by setting $r_{z}=0$ in Eq. (11) and (2). Fig. 2 (d) and (e), and Fig. 3 (d) and (e) show the projection distances of the corresponding DTOCS and WDTOCS images. Dividing the DTOCS or WDTOCS distance values with the corresponding projection distances result in values indicating the average height variation along the shortest path to the nearest reference pixel.

\section{Distance and Nearest Neighbor Transformation}

An efficient priority pixel queue transformation algorithm for calculating the DTOCS is presented in [14]. It is very similar to the Fast Marching algorithm for calculating forward propagating level sets [15], but the calculations are simpler, as the DTOCS algorithm is developed directly for the discrete geometry inherent for digital image processing. The reference pixels, from which distances are calculated, are enqueued into a minimum heap, from which they are dequeued in priority order. New distance values are calculated for neighbors of the dequeued pixel, and subsequently enqueued. The best first approach ensures that distance values are final when they are dequeued, and propagated further. The projection distance values are calculated simultaneously with the DTOCS or the WDTOCS values. The propagation order also enables easy implementation of the nearest neighbor transformation, which assigns the identity of the nearest feature pixel to each pixel in the image. The distances and the nearest site are determined according to the DTOCS, as described in [16]. Unique seed values assigned to each reference pixel are propagated simultaneously with the 
distance values, so that each pixel gets the seed value of the pixel from which the distance propagated to it. A similar region growing algorithm for tessellation of 3D volumes is presented in [17. The complexity of the pixel queue algorithm is in $\mathcal{O}\left(n \log n_{q}\right)$, where $n_{q}$ is the length of the queue, which varies throughout the transformation. As $n_{q} \ll n$, the algorithm is near-linear, with running times increasing only slightly with increasing surface complexity [14.

\section{Roughness Measurement Using the DTOCS}

In the new roughness evaluation method, a distance map is calculated using an evenly spaced set of reference pixels, or seeds, on the original image. A nearest neighbor transform is calculated simultaneously to attach each pixel to the nearest reference pixel. The curved distances within each region are divided by the corresponding projection distances. The more variation there is around the seed pixel, the larger are the distances. The averages of the normalized distance values within each region produce a roughness map of local roughness values.

Fig. [4illustrates how the roughness measurement method proceeds on a topography image. The original surface image, Fig. 4 (a), is 250 pixels wide and 50 pixels high, but represents a square surface, a piece of paper of size $2.5 \mathrm{~mm} \times 2.5 \mathrm{~mm}$.

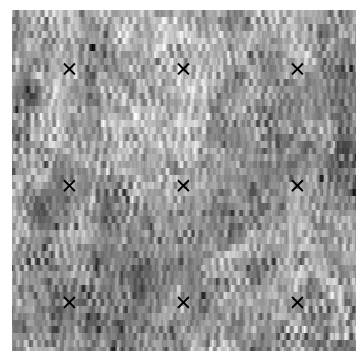

(a) Original image, seeds

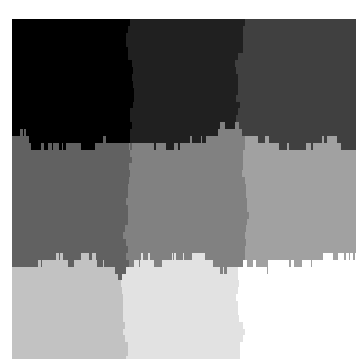

(d) NNT image

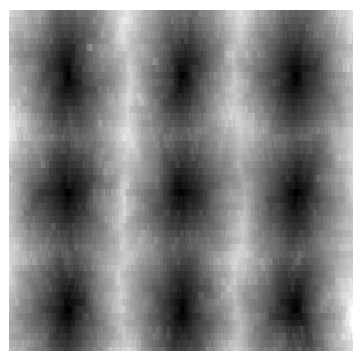

(b) DTOCS image

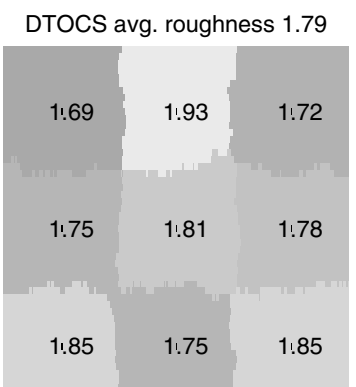

(e) roughness map

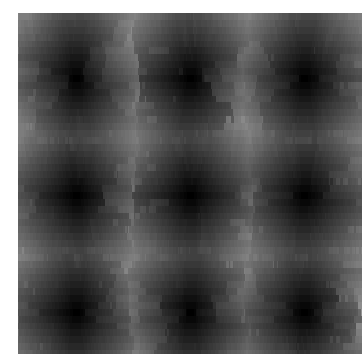

(c) Proj. distance image

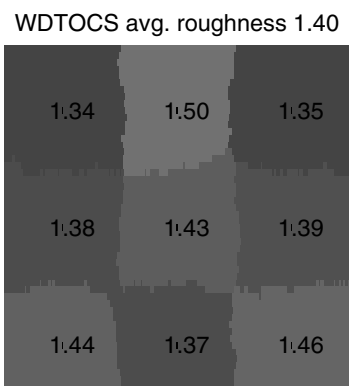

(f) roughness map

Fig. 4. The phases in the roughness measurement method (a)-(e). Image (f) shows that WDTOCS roughness values are consistently smaller than DTOCS roughness values. 
This means that one pixel represents a surface area of size $10 \mu \mathrm{m} \times 50 \mu \mathrm{m}$. The height values are measured in micrometers, but as the height variation is very small compared to the horizontal displacements, a factor 10 is added to the height component to emphasize the variation. The resulting scaling factors for the local distances are $r_{x}=10, r_{y}=50$, and $r_{z}=10$, or in practise, $r_{x}=1, r_{y}=5$, and $r_{z}=1$.

As local distances based on gray-values can vary significantly, the nearest neighbor transformation can result in any shapes of regions around each site. The region sizes also vary, as the distance propagation covers more pixels in a smoother area. Seed pixels in areas with higher variation are typically surrounded by smaller nearest neighbor regions. On a highly varying surface, some seed values may not propagate at all, if each neighbor of the seed pixel is closer, or equally close, to one of the surrounding reference pixels. In such cases, the roughness value is approximated using the average of the distance values in the 8 -neighborhood of the seed pixel, plus one. The idea is that distances from the reference pixel to its neighbors must be greater or equal to the distance between each neighbor and some other reference pixel. This approximation can make the method more robust against noise, as a reference pixel differing significantly from its neighborhood can "borrow" its roughness value from its neighborhood rather than cause a peak in the roughness map.

\section{Roughness Properties}

Surface roughness can consist of different scales of variations, as demonstrated by the example surfaces in Fig. 5. Synthetic surfaces 2, 3 and 4 are very similar when examined at close range, as they are created by adding the same noise component to a flat surface, and to surfaces with larger scale Gaussian variations, or bumps. It is obvious that surface 2 is smoother than surfaces 3 and 4 , and surface 1 with less local variation even smoother. Surface 5 is locally very smooth, but the larger scale variation is similar as in surface 4. Surfaces 3-5 demonstrate so called waviness 4, whereas the term roughness refers to the local variation present in surfaces 1-4. The third roughness property defined in [4 is called form, and refers to non-frequency components of the surface topography, which for paper surfaces should be a flat plane. The first four surfaces in Fig. 5 are clearly in order of increasing roughness. Surface 5 is smoother than surface 4, but comparison with surface 3 depends on whether waviness or local roughness is more significant. Alternatively, roughness properties can be classified into three different roughness classes: 1) Optical roughness at length scales $<1 \mu \mathrm{m}, 2$ ) Micro roughness at $1 \mu \mathrm{m}-100 \mu \mathrm{m}$ and 3) Macro roughness at $0.1 \mathrm{~mm}-1$ $\mathrm{mm}$. All these three roughness classes affect paper gloss, and micro and macro roughness also paper uniformity [18].

Roughness is usually defined as a deviation from an ideal, flat reference plane, where all the surface elements are in the same level. Our distance transform approach is designed for measuring local or smaller scale roughness, and additional measures will be needed to extract waviness properties. In the experiments, the DTOCS roughness measure is compared to the arithmetic mean deviation, $S_{a}$, 


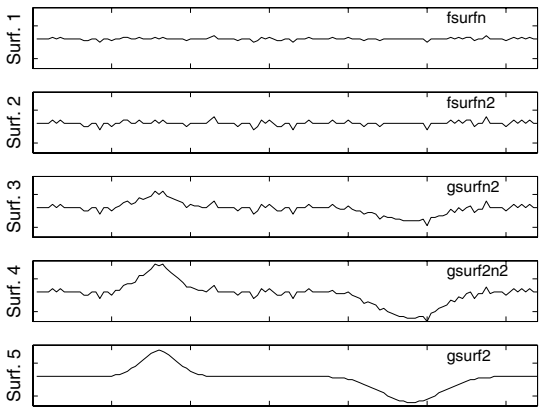

Fig. 5. Profiles of example surfaces with different roughness properties

calculated from the so called roughness surface, from which the effect of the waviness has been removed by filtering. The measured topographies are filtered using a Gaussian filter in order to extract roughness and waviness surfaces from the inspected surface. The result of low-pass Gaussian filtering is the waviness surface, and the roughness surface is extracted from the original surface by subtracting the waviness surface from the original surface, see Fig. 6. The filter is calculated by a direct convolution of the surface topography with a Gaussian weighting function $S(x, y)$, which is given by

$$
S(x, y)=\frac{1}{\beta \lambda_{x c} \lambda_{y c}} \exp \left\{-\frac{\pi}{\beta}\left[\left(\frac{x}{\lambda_{x c}}\right)^{2}+\left(\frac{y}{\lambda_{y c}}\right)^{2}\right]\right\},
$$

where $x$ and $y$ are the positions from the center of the weighting function, $\left(\lambda_{x c}, \lambda_{y c}\right)$ are the cutoff wavelengths at $50 \%$ attenuation ratio and $\beta=\ln 2 / \pi[4$. ISO standards recommend cutoff wavelengths $0.08,0.25,0.8,2.5$, and $8 \mathrm{~mm}$ [19].

The roughness surface can be characterized by statistical analysis, 2-D spectral analysis and time series analysis. A statistical roughness parameter, the arithmetic mean deviation of the surface, $S_{a}$, is defined as

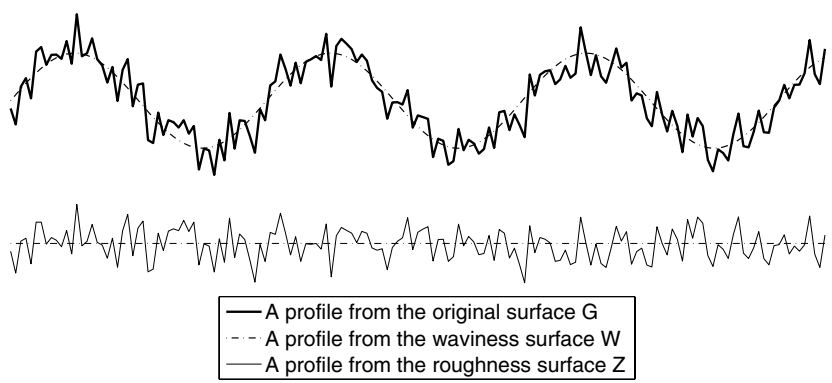

Fig. 6. Profiles from the original, the waviness and the roughness surface 


$$
S_{a}=\frac{1}{N_{x} N_{y}} \sum_{i=1}^{N_{y}} \sum_{j=1}^{N_{x}}\left|Z\left(x_{j}, y_{i}\right)\right|,
$$

where $N_{x}$ and $N_{y}$ are the number of data points in the $x$ - and $y$-direction and $Z(x, y)$ is the height value of the roughness surface in point $(x, y)$. The $S_{a}$ parameter is very commonly used in practical applications 4], so obtaining high correlations between the DTOCS roughness measure and $S_{a}$ indicates that the new roughness evaluation method can be useful in practice.

\section{Experiments}

The roughness of synthetic images, some of which were used as examples in Fig. 5, were evaluated using the DTOCS and the WDTOCS. The nine original surfaces in Fig. 7a) are arranged so that the waviness increases from top to bottom. The first surface is flat, the second one contains Gaussian bumps, and in the third surface the Gaussian bumps are twice as high or deep. The local roughness increases from left to right, so surfaces to the left are locally smooth, surfaces in the middle contain a noise component, and to the right, the noise component is doubled. The standard deviation of surface height values is indicated above each image. Fig. 7 (b) shows the DTOCS roughness maps of the corresponding test surfaces. The WDTOCS roughness maps, not shown due to lack of space, are visually similar, but with consistently lower roughness values. The intensity of each region indicates the local roughness value, that is, darker regions lie in smoother areas of the image. Areas with only local roughness without waviness can have equally high DTOCS roughness values as areas containing Gaussian

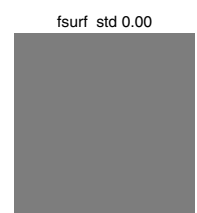

gsurf std 1.40

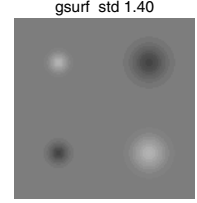

gsurf2 std 2.79

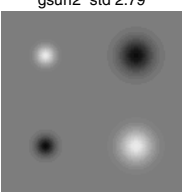

(a) Original surface images

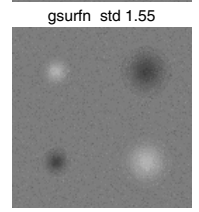

gsurf2n std 2.87 gsurfn2 std 1.95

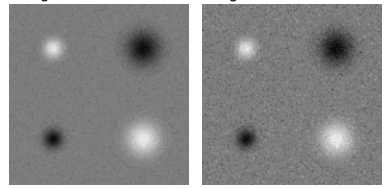

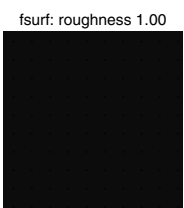
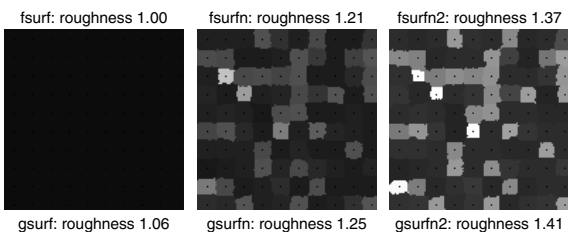

gsurfn: roughness 1.25

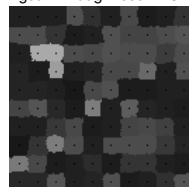

gsurfn2: roughness 1.41

gsurf: roughness 1.06

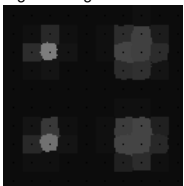

gsurf2: roughness 1.11

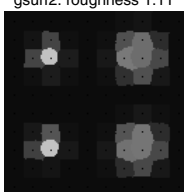

gsurf2n: roughness 1.30
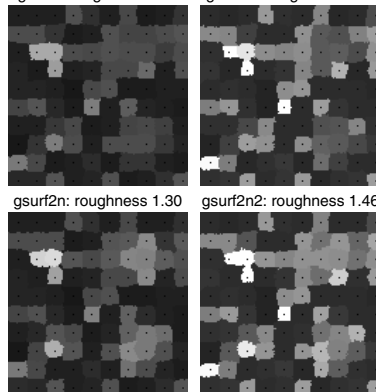

gsurf2n2: roughness 1.46

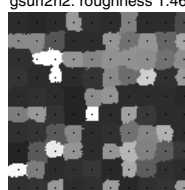

(b) DTOCS roughness maps

Fig. 7. Synthetic images and their roughness maps based on a $10 \times 10$ grid of seeds 

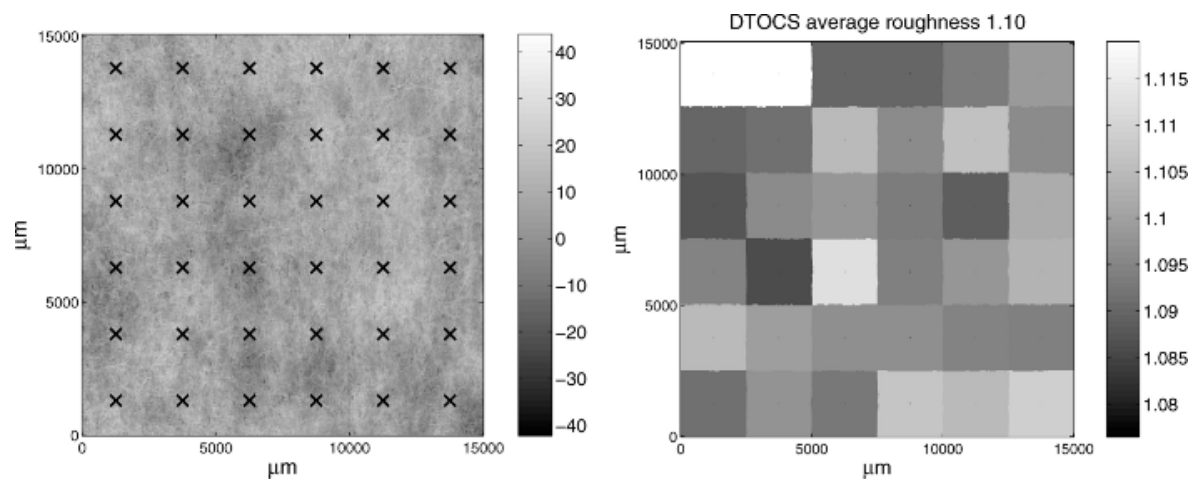

Fig. 8. A sample from test set C, and its DTOCS roughness map
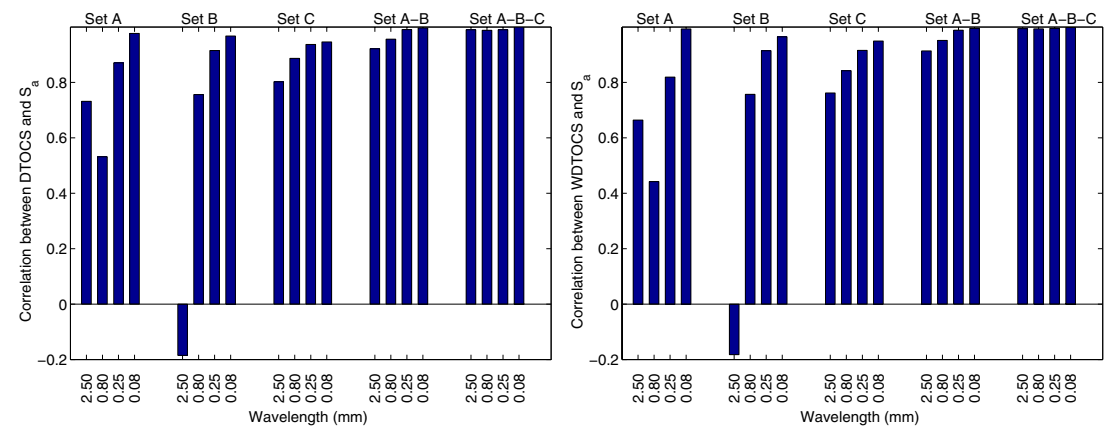

Fig. 9. Correlation values for comparison between $S_{a}$ and DTOCS or WDTOCS

bumps, as a smooth slope produces only slightly larger distance values than a flat surface. The average roughness values shown above each surface image increase only slightly with increasing waviness.

Experiments on real profilometer data were performed using sample sets, which include a variety of paper samples and cardboard samples. Sample set A consists of 8 light weight coated paper samples, sample set B of 11 supercalendered paper samples and sample set $\mathrm{C}$ of 8 base cardboard samples. The first two sample sets, A and B are similar in roughness compared to set C, which is significantly rougher. Each sample was marked with a $15 \mathrm{~mm} \times 15 \mathrm{~mm}$ measurement area, on which the profilometer measurements were performed. The resolution in the $x$-direction is $10 \mu \mathrm{m}$ and the resolution in the $y$-direction $50 \mu \mathrm{m}$. The height value is given in micrometers, that is, $r_{x}=10, r_{y}=50$ and $r_{z}=1$. One sample from the roughest test set $\mathrm{C}$ is shown in Fig. 8. visualizing also the $6 \times 6$ grid of seed pixels used in the experiments. It can be seen that the roughness variation is very small within the image, resulting in almost square regions in the roughness map. The variation in the corresponding WDTOCS roughness map is even smaller. This is due to the fact that the height variation in the profilometer data is small compared to the resolution in the $x$-direction, 


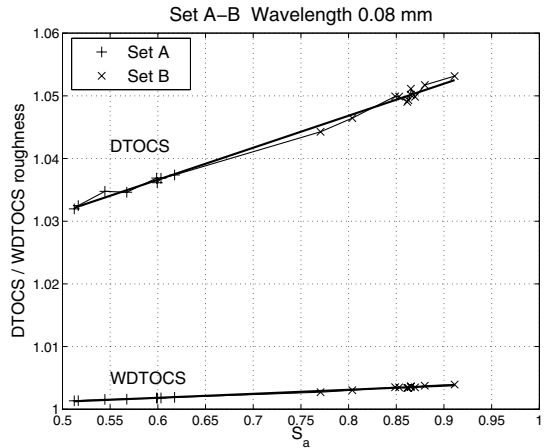

(a) Combined set of $\mathrm{A}$ and $\mathrm{B}$

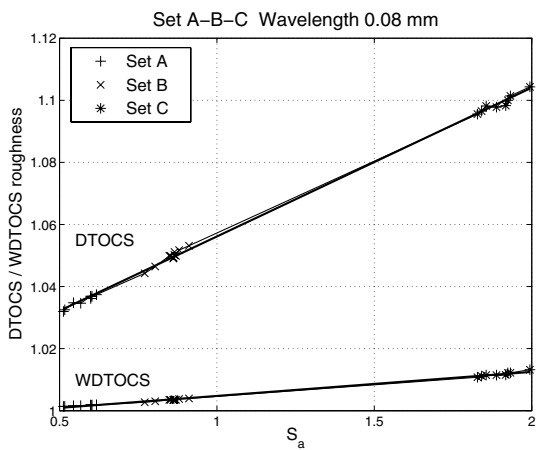

(b) Set including sets A, B and C

Fig. 10. Scatter plots of $S_{a}$ and DTOCS or WDTOCS roughness values for a combined test set including set $\mathrm{A}$ and set $\mathrm{B}$, and for a test including sets $\mathrm{A}, \mathrm{B}$ and $\mathrm{C}$

and especially compared to the resolution in the $y$-direction. The normalization with the corresponding projection distance makes the effect of the variation in the $y$-direction almost negligible. A scaling factor for the height variation, as in the example shown in Fig. 4. may be introduced in future works to emphasize the height variation.

The results in Fig. 9 show high correlations between the DTOCS or the WDTOCS roughness measure, and the arithmetic mean deviation $S_{a}$. The results are good for the micro roughness wavelength $0.08 \mathrm{~mm}$, and relatively good for the macro roughness wavelengths $0.25 \mathrm{~mm}$ and $0.8 \mathrm{~mm}$. The filtering wavelength $2.5 \mathrm{~mm}$ approaches the size of the measurement area, so the resulting $S_{a}$ value does not characterize the surface accurately. The correlations calculated using the micro roughness wavelength are strong for all tests sets A, B and C separately, and also for a combined set containing the samples from A and B, and a set containing all samples from A, B and C. Since the samples in set C are clearly rougher than the samples in A and B, the correlation is very strong, but it can been seen from Fig. 10 (b) that there is a clear linear dependency between the DTOCS or WDTOCS roughness values, and the $S_{a}$ roughness parameter calculated using the micro roughness wavelength $0.08 \mathrm{~mm}$. Fig. 10 also illustrates, that the DTOCS roughness values are consistently higher than the WDTOCS roughness values, as the DTOCS local distance overestimates the locally Euclidean distance between neighbor pixels.

\section{Discussion}

A new distance transform method has been developed for measuring surface roughness. The distance transforms, the DTOCS and the WDTOCS, which measure distances along a surface, have been generalized to anisotropic grids. Previously, the distance transforms have been applied to gray-level images, but the new modifications are applicable to floating point altitude data. In the new 
roughness measurement method, the distance transforms are combined with a nearest neighbor transform to produce a roughness map characterizing local roughness within regions of the image. Experiments were performed on synthetic surfaces, and on real topographies of paper surfaces obtained using a profilometer. The correlations between the arithmetic mean deviation of the surface, $S_{a}$, for the micro roughness wavelength, and the new methods were strong for all test sets. The results suggest that the roughness can be calculated directly from the topography using the DTOCS or the WDTOCS, without first extracting the roughness surface using Gaussian filtering. Further experiments and comparisons to other methods will be performed in future work.

In future work, the distance transform method will be tested using different numbers of seed pixels for the nearest neighbor transform. Alternative configurations, like a hexagonal grid of seeds, may also be beneficial compared to the square grid used here. Also, more tests are needed to determine whether the WDTOCS method provides more accurate results compared to the DTOCS, which is slightly simpler and faster to calculate. Furthermore, as it is well known that the piecewise Euclidean distance overestimates true distances (see e.g. [20]), the more accurate Optimal DTOCS [8] will be generalized to images of unequal resolution, for example, by using weights derived by Sintorn and Borgefors [12 for distance transforms of binary images in rectangular grids. Alternatively, ideas behind weighted 3D distance transforms for elongated voxel grids [13] could be utilized in order to obtain more accurate approximations of true distances along anisotropic gray-level surfaces. However, in measuring surface roughness, using the most accurate approximations of true distances may not be necessary, as long as the distance values increase in proportion with the surface variation. As shown by the experiments, the effect of the height variation may need to be emphasized by using a scaling factor for the height component of the local distance. In this work, the distance values approximate the true distance measured along the representation of the surface. However, the representation is not entirely accurate, as the sparse $50 \mu \mathrm{m}$ resolution data misses some small scale variation in the surface. In future work, data measured at the more dense resolution, $10 \mu \mathrm{m}$, in both directions will be available for comparison.

This work is part of a research project aiming to develop a machine vision system for measuring the roughness of paper, which could be used in paper industry during processing. The new method was shown to be a simple and efficient approach to characterize the micro roughness of paper surfaces. Particularly, the roughness maps, which provide interesting information about the roughness properties of different areas of the surface, will be investigated further. If the DTOCS roughness values and the $S_{a}$ values calculated from the same nearest neighbor regions are found to correlate, the DTOCS provides an easy way to divide the surface to areas with different roughness properties. The distance transform measurements could be part of a larger pattern recognition system, providing features to be used together with, for example, fractal dimension or statistical features in classifying any surfaces, not just paper, by their roughness. 


\section{References}

1. Wagberg, P., Johansson, P. A.: Surface profilometry - a comp. between optical and mechanical sensing on printing papers. Tappi Journal 76(12) (1993) 15-121

2. Toivanen, P.: New geodesic distance transforms for gray-scale images. Pattern Recognition Letters 17 (1996) 437-450

3. Johansson, J-O.: Measuring homogenity of planar point-patterns by using kurtosis. Pattern Recognition Letters 21(13-14) (2000) 1149-1156

4. Stout, K. J. (ed.): Development of Methods for the Characterization of Roughness in Three Dimensions. Penton Press, revised reprint (original 1993) edition (2000)

5. Yuan, C., Peng, Z., Yan, X.: Surface characterization using wavelet theory and confocal laser scanning microscopy. Journal of Tribology 127(2) (2005) 394-404

6. Kent, H. J.: The fractal dimension of paper surface topography. In: TAPPI/CPPA International Printing and Graphics Arts Conference, Vancouver, Canada (1990) $73-78$

7. Kuparinen, T., Rodionov, O., Toivanen, P., Mielikainen, J., Bochko, V., Korkalainen, A., Parviainen, J., Vartiainen, E.: Fractal dimension analysis and statistical processing of paper surface images towards surface roughness measurement. In: Scandinavian Conference on Image Analysis (SCIA) Joensuu, Finland (2005) $1218-1227$

8. Ikonen, L., Toivanen, P.: Shortest routes on varying height surfaces using gray-level distance transforms. Image and Vision Computing 23(2) (2005) 133-141

9. Borgefors, G.: On digital distance transforms in three dimensions. Computer Vision and Image Understanding 64(3) (1996) 368-376

10. Ikonen, L.: Distance Transforms on Gray-Level Surfaces. PhD thesis, Lappeenranta University of Technology (2006)

11. Ikonen, L., Toivanen, P.: Distance and nearest neighbor transforms of gray-level surfaces. Submitted to Pattern Recognition Letters, September (2005)

12. Sintorn, I-M., Borgefors, G.: Weighted distance transforms in rectangular grids. In: International Conference on Image Analysis and Processing (ICIAP), Palermo, Italy (2001) 322-326

13. Sintorn, I-M., Borgefors, G.: Weighted distance transforms for images using elongated voxel grids. In: Discrete Geometry for Computer Imagery (DGCI), Berlin, Germany (2002) 244-254

14. Ikonen, L.: Pixel queue algorithm for geodesic distance transforms. In: Discrete Geometry for Computer Imagery (DGCI), Poitiers, France (2005) 228-239

15. Sethian, J. A.: Level Set Methods and Fast Marching Methods. Cambridge University Press, 2nd edition (1999)

16. Ikonen, L., Toivanen, P.: Distance and nearest neighbor transforms of gray-level surfaces using priority pixel queue algorithm. In: Advanced Concepts for Intelligent Vision Systems (ACIVS), Antwerp, Belgium (2005) 308-315

17. Kapoutsis, C. A., Vavoulidis, C. P., Pitas, I.: Morphological iterative closest point algorithm. IEEE Transactions on Image Processing 8(11) (1999) 1644-1646

18. Niskanen, K. (ed.): Paper Physics. Book 16 - Papermaking Science and Technology. Fabet Oy and Tappi Press, Jyväskylä (1998)

19. National Institute of Standards and Technology. Internet-based surface metrology algorithm testing system. WWW-page. Available from http://ats.nist.gov/VSC/jsp/index.jsp, accessed 2006-03-31

20. Borgefors, G.: Distance transformations in digital images. Computer Vision, Graphics, and Image Processing 34 (1986) 344-371 